\title{
Doping dependence of the Néel temperature in Mott-Hubbard antiferromagnets: Effect of vortices
}

\author{
Carsten Timm and K.H. Bennemann \\ Institut für Theoretische Physik, Freie Universität Berlin, Arnimallee 14, D-14195 Berlin, Germany
}

(October 18, 2018)

\begin{abstract}
The rapid destruction of long-range antiferromagnetic order upon doping of Mott-Hubbard antiferromagnetic insulators is studied within a generalized Berezinskii-Kosterlitz-Thouless renormalization group theory in accordance with recent calculations suggesting that holes dress with vortices. We calculate the doping-dependent Néel temperature in good agreement with experiments for high- $T_{c}$ cuprates. Interestingly, the critical doping where long-range order vanishes at zero temperature is predicted to be $x_{c} \sim 0.02$, independently of any energy scales of the system.
\end{abstract}

74.72.-h, 75.70.Ak, 05.10.Cc, 75.50.Ee

The study of lightly doped Mott-Hubbard (MH) antiferromagnetic insulators is of great current interest, since the insulating parent compounds of cuprate high- $T_{c}$ superconductors are of this type. The various parts of the phase diagram of these compounds are believed to be intimately related. Therefore it is important to understand the properties of the antiferromagnetic phase, in particular the rapid destruction of magnetic order upon doping and the anomalously small critical doping of $x_{c} \sim 0.02$ holes per copper ion [1]. In the present letter we derive the Néel temperature $T_{N}$ as a function of doping using a generalized Berezinskii-Kosterlitz-Thouless (BKT) renormalization group theory [2,3] for the vortices in the antiferromagnetic state. There are two types of vortices, thermally created electrically neutral ones and electrically charged ones, which are centered at the holes. Both types are nucleated separately (as vortex-antivortex pairs), but additively screen the vortex interaction, with a common unbinding temperature $T_{N}(x)$. This temperature is indeed strongly reduced upon doping and vanishes at $x_{c} \sim 0.02$ independently of the energy scales of the system. Our approach is independent of any particular microscopic model and can thus serve as a guide for electronic theories.

Our physical picture is the following: The holes introduced by doping are mainly located at the planar oxygen sites, where they frustrate the antiferromagnetic exchange interaction between copper spins due to their tendency to form copper-oxygen spin singlets 四, 死. This may lead to ferromagnetic coupling between the two spins [6]. Since the system is approximately twodimensional, the staggered magnetization can form a vortex as sketched in Fig. 1 to evenly distribute the frustration induced by the hole. On the other hand, neutral vortices without a hole in their core can be thermally created. Due to the easy-plane Dzyaloshinskii-Moriya anisotropy [7], the staggered magnetization can be described by a two-component order parameter at low energies, leading to a logarithmic size dependence of the single-vortex energy [8,9]. This implies a logarithmic vortex interaction, making BKT scaling ideas applica- ble. To describe the interplay of charged and neutral vortices, which determines the Néel temperature $T_{N}$, we have to extend the BKT theory to a system with two kinds of topological defects. One important point is that the density of charged vortices is given by the doping $x$. The other is that we have to describe the screening of the vortex interaction due to both types.

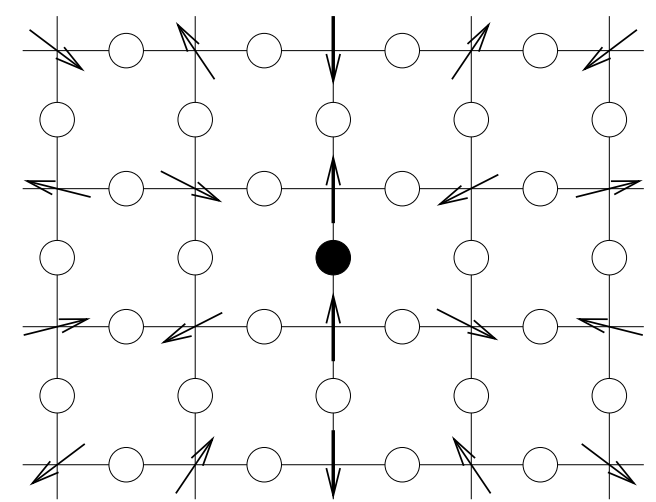

FIG. 1. Schematic representation of a charged vortex induced by a single hole at an oxygen site (black circle). The hole frustrates the interaction between copper spins (arrows). The circles denote oxygen atoms. A neutral vortex would be centered on the square between four oxygen atoms and not have a hole in its core.

Electronic theory supports our picture: Within unrestricted Hartree-Fock theory Vergés et al. [8] found several competing low-energy configurations for a lightly hole-doped Hubbard model, including spin polarons, domain walls, and holes dressed with vortices and antivortices. In a spin polaron the staggered local moments in the vicinity of the hole are reduced but still collinear, while in a vortex (antivortex) they rotate through $2 \pi$ $(-2 \pi)$. Seibold 10 using a slave-boson approach and Berciu and John [9] within a self-consistent Hartree-Fock theory found that an even number of holes dress with vortices and antivortices (or merons [9]) in the ground state for appropriate parameters. Since the energy of a single vortex diverges (logarithmically [3,8,961) with system size, whereas that of a vortex-antivortex pair remains finite, 
only pairs are created in infinite systems.

An advantage of BKT-type theories is that they include fluctuations on all length scales, in particular on large ones, which are crucial close to the phase transition. Previous studies that only included fluctuating local moments without correlations between them overestimated the critical doping $x_{c}$ 11. Similarly, the decrease of $T_{N}$ with $x$ obtained from the fluctuation exchange approximation is also too slow [12]. By including correlations between neighboring spins into a slave-boson theory for the three-band Hubbard model, Schmalian et al. [5] obtained a critical doping of $x_{c} \sim 0.025$ in better agreement with experiment. However, this approach takes only fluctuations on the length scale of the lattice constant into account, but neglects fluctuations on larger length scales.

In BKT theory the interaction of vortices is screened by the polarization of vortex pairs lying between them [2.3. As the temperature is increased more pairs are thermally created leading to increased screening. At the Néel temperature the screening becomes strong enough for the largest pairs to break up. The resulting free vortices destroy the magnetic order. The situation here is more complex: First, we have to take account of the screening due to both charged and neutral vortices, and second, the density of charged vortices is fixed by the doping level $x$.

Only neutral pairs are thermally created, whereas charged vortices enter the system only upon doping. In principle one could imagine a single hole doped into the system to form a pair consisting of a charged vortex and a neutral antivortex or vice versa, but microscopic calculations 8, 10,9] do not find this configuration at $T=0$. Rather, two holes are needed to produce a vortex-antivortex pair. For simplicity we assume this to hold also at finite temperatures. However, even if mixed pairs are not created upon doping, they are formed when vortex pairs exchange partners.

The density of neutral vortices is controlled by their chemical potential $\mu_{\text {neu }}$ or equivalently the vortex core energy $E_{\text {core }}=-\mu_{\text {neu }}$, which depends on details of the copper-oxygen and copper-copper interactions and is treated here as a parameter. The energy of a vortexantivortex pair is $2 E_{\text {core }}+V$ with the interaction 13 $V(r)=q^{2} \ln \left(r / r_{0}\right)$, where $q^{2}=2 \pi J S^{2}$ is the strength of the interaction, $J$ is the exchange interaction between nearest neighbors, $S=1 / 2$ is the spin, and $r_{0}$ is the small-distance cutoff of BKT theory. $r_{0}$ can be interpreted as the smallest possible vortex-antivortex separation [2:3]. Two charged vortices additionally experience a Coulomb interaction, which, however, is irrelevant in the renormalization-group sense, since it falls off faster than $V(r) \sim \ln r$.

The probability of creating a neutral or charged vortex in an area $r_{0}^{2}$ is given by its fugacity $y_{\text {neu }}$ and $y_{\mathrm{ch}}$, respectively. Since we have assumed that vortices are only created in neutral or charged pairs, we consider the pair fugacities $y_{\text {neu }}^{2}$ and $y_{\mathrm{ch}}^{2}$. For the smallest possible neutral pairs of size $r_{0}$,

$$
y_{\text {neu }}^{2}\left(r_{0}\right)=C_{\text {neu }}^{2} e^{2 \beta \mu_{\text {neu }}},
$$

where $C_{\text {neu }}$ is a constant of order unity [2] and $\beta$ is the inverse temperature. The constraint on the density of charged vortices is implemented by choosing $y_{\mathrm{ch}}^{2}\left(r_{0}\right)$ in such a way that their total density equals the hole density, see below. The vortex interaction is screened by the polarization of smaller vortex pairs, $V(r)=$ $q^{2} / \epsilon(r) \ln \left(r / r_{0}\right)$. The screening is described by the spinwave stiffness $K(r)=\beta q^{2} / 2 \pi \epsilon(r)$. In the renormalization group, small pairs of sizes between $r$ and $r+d r$ are integrated out and their effect is approximately incorporated into renormalized quantities $K(r), y_{\text {neu }}^{2}(r)$, and $y_{\mathrm{ch}}^{2}(r)$. Starting from $r=r_{0}$, this operation is repeated for larger and larger pairs leading to the recursion relations [2.14]

$$
\begin{aligned}
d y_{\eta}^{2} / d l & =2(2-\pi K) y_{\eta}^{2}, \\
d K / d l & =-4 \pi^{3}\left(y_{\text {neu }}^{2}+y_{\text {ch }}^{2}\right) K^{2} .
\end{aligned}
$$

The initial conditions are Eq. (11) and $K\left(r_{0}\right)=\beta q^{2} / 2 \pi$. Equation (2) determines the fugacities of neutral $(\eta=$ neu) and charged $(\eta=\mathrm{ch})$ pairs of size $r=r_{0} e^{l}$. Two separate equations for $y_{\text {neu }}^{2}$ and $y_{\mathrm{ch}}^{2}$ are present, since we assume that vortices are created either as neutral pairs or as charged pairs with two holes. Both types feel the same screened interaction $V$ at large distances so that the same stiffness $K$ appears. Differences at smaller separation are incorporated into the core energies. Equation (3) describes the additional screening due to pairs of size $r_{0} e^{l}$. Their total density is proportional to $y_{\text {neu }}^{2}+y_{\mathrm{ch}}^{2}$.

If the stiffness $K$ vanishes for $l \rightarrow \infty$, the interaction is fully screened for large pairs $(\epsilon \rightarrow \infty)$, which thus become unbound, destroying the magnetic order. Since the interaction on large length scales is the same for neutral and charged vortices, this unbinding happens at a single transition for both types. While solving Eqs. (2) and (3) we have to simultaneously satisfy the constraint on the density $n_{\mathrm{ch}}$ of charged vortex pairs. As shown in Ref. [15], this density can be expressed in terms of the fugacity,

$$
n_{\mathrm{ch}}=\int_{r_{0}}^{\infty} d r 2 \pi r \frac{y_{\mathrm{ch}}^{2}(r)}{r^{4}}=\frac{2 \pi}{r_{0}^{2}} \int_{0}^{\infty} d l e^{-2 l} y_{\mathrm{ch}}^{2}(l) .
$$

The pair density has to equal half the density of holes, $n_{\mathrm{ch}}=x / 2 a^{2}$, where $a$ is the lattice constant. In practice, the recursion relations are integrated numerically to find $y_{\mathrm{ch}}^{2}(l)$, from which we calculate $n_{\mathrm{ch}}$. The initial value $y_{\mathrm{ch}}^{2}(0)$ is varied until the contraint is satisfied.

The resulting phase diagram is shown in Fig. 2. We used $C_{\text {neu }}=1, J=1800 \mathrm{~K}$, and $r_{0}=2 a$, and varied the core energy $E_{\text {core }}$. The phase below the Néel temperature $T_{N}$ shows quasi-long-range antiferromagnetic order, which is made long range by the weak interlayer 
exchange. The phase for $T>T_{N}$ or $x>x_{c}$ is characterized by free vortices, which destroy the long-range order, but leave short-range order on the length scale of the separation between free vortices intact. Short range correlations have indeed been observed in cuprates up to much larger dopings. For $T \rightarrow 0$ we find the critical pair density,

$$
n_{\mathrm{ch}}^{c} \approx 0.04273 r_{0}^{-2} .
$$

The numerical factor is universal: Since neutral vortices do not exist for $T \rightarrow 0$, it cannot depend on $E_{\text {core }}$ and $C_{\text {neu }}$. The remaining energy scale $q^{2}$ does not enter the result, since it is multiplied by the diverging $\beta=1 / k_{B} T$. While $n_{\mathrm{ch}}^{c}$ and thus the critical doping $x_{c}=2 n_{\mathrm{ch}}^{c} a^{2}$ are independent of energy scales, they do depend on the nonuniversal minimal separation $r_{0}$ of two vortices which is of the order of twice the core radius or twice the correlation length. Slave-boson and Hartree-Fock calculations 10,9] show that the core radius is not significantly larger than a lattice spacing $a$. In Fig. 2 we have taken the core radius to be $a$ so that $r_{0}=2 a$, which results in $x_{c} \sim 0.021$ in very good agreement with experiments on $\mathrm{La}_{2-x} \mathrm{Sr}_{x} \mathrm{CuO}_{4}$ [1, 16 [18]. For $\mathrm{Y}_{1-z} \mathrm{Ca}_{z} \mathrm{Ba}_{2} \mathrm{Cu}_{3} \mathrm{O}_{6}$ experiments find $z_{c} / 2 \sim 0.03$ [18], of the same order of magnitude as our value of 0.021 (the number of holes per copper atom is $z / 2$ in this double-layer compound). For $\mathrm{YBa}_{2} \mathrm{Cu}_{3} \mathrm{O}_{7-\delta}$ a critical hole concentration of 0.021 corresponds to $\delta_{c} \sim 0.68$, following Tallon et al. [19], in good agreement with experiments 20].

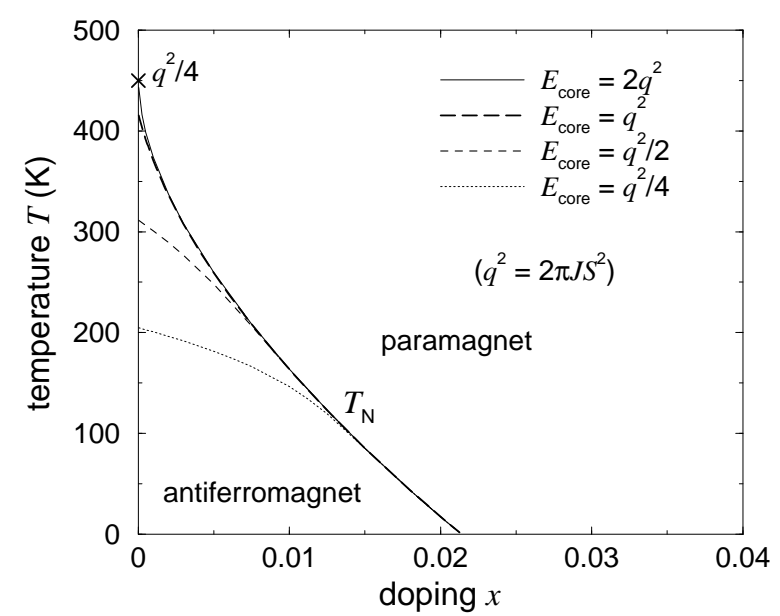

FIG. 2. Phase diagram for the antiferromagnetic phase of the cuprates. The Néel temperature $T_{N}$ is calculated using four values of the core energy $E_{\text {core }}$ of thermally created vortices. Note that the curves for larger $E_{\text {core }}$ are very close to the one for $E_{\text {core }}=2 q^{2}$. The symbol $\times$ at zero doping denotes the maximal possible transition temperature.

In the high-temperature region of the phase diagram the overall temperature scale is set by the maximal possible Néel temperature $T_{N}^{\max }=q^{2} / 4=\pi J S^{2} / 2$. For $S=1 / 2$ this gives $T_{N}^{\max } \approx 0.393 J$, compared to the mean-field result $T_{N}^{\mathrm{mf}}=0.5 \mathrm{~J}$ for a Heisenberg antiferromagnet on a cubic lattice with interlayer exchange $J_{\perp} \ll J$. The reduction of $T_{N}$ is due to fluctuations, which are strong for quasi-two-dimensional systems. The actual value of $T_{N}(x=0)$ and the shape of the curve $T_{N}(x)$ at small doping are determined by the core energy $E_{\text {core. }}$ Note, we obtain the correct temperature scale under the reasonable assumption that $E_{\text {core }}$ is not very much smaller than the interaction strength $q^{2}$. Experimentally, $T_{N}$ is found to depend only weakly on doping for small $x$ [17], which requires a small core energy. Then many neutral vortices are created at a given temperature so that charged vortices only become relevant at higher doping. Conversely, for large core energy only a few thermal vortices are present even at $T_{N}^{\max }$. For $E_{\text {core }} \gtrsim 2 q^{2}$ the curve $T_{N}(x)$ in Fig. 2 does not change appreciably with $E_{\text {core }}$ so that the charged vortices would determine the magnetic properties even at very small doping.

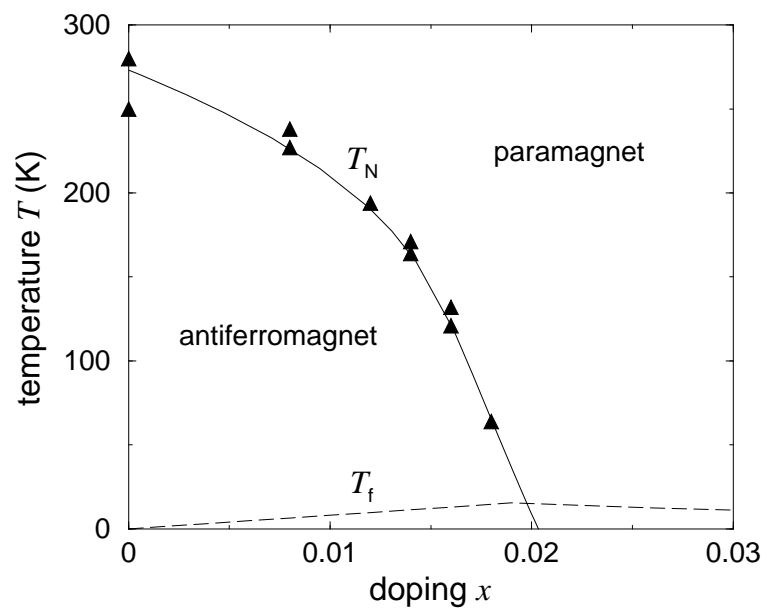

FIG. 3. Comparison of the calculated Néel temperature for $E_{\text {core }} / q^{2} \approx 0.13$ (solid line) and results for $T_{N}$ from NQR and $\mu \mathrm{SR}$ experiments for $\mathrm{La}_{2-x} \mathrm{Sr}_{x} \mathrm{CuO}_{4}$ (triangles) [17]. The dashed line schematically shows the freezing temperature $T_{f}$ [18], below which the holes become immobile.

Quantitative agreement with $\mu \mathrm{SR}$ and NQR experiments on $\mathrm{La}_{2-x} \mathrm{Sr}_{x} \mathrm{CuO}_{4}$ by Borsa et al. 117] can be obtained by appropriate choices of the exchange $J$, the core energy $E_{\text {core }}$, and the core radius $r_{0}$, see Fig. 3 . For this plot, $J=2410 \mathrm{~K}, E_{\text {core }}=0.1303 q^{2}=493 \mathrm{~K}$, and $r_{0}=2.052 a$. Typical experimental values are $J \sim$ $1400 \mathrm{~K}$ [21]. This discrepancy may be due to the simplified description of the anisotropic antiferromagnet by two-component spins or to the neglect of the interlayer exchange $J_{\perp}$ and the doping dependence of $J$.

We now briefly comment on electron-doped cuprates. While we reproduce the correct critical doping for holedoped compounds, our approach would not yield the much larger critical doping $x_{c, e} \sim 0.14$ in electron-doped cuprates [1]. The reason is that the additional electrons mainly fill up the copper $3 d$ orbitals and destroy the mag- 
netic moments at the copper sites. Thus, the main effect of electron doping is to dilute the antiferromagnet. There is no spin-singlet formation involved and hence no tendency towards vortex formation. Then differently as in the case of hole doping $T_{N}$ decreases due to spin dilution.

The validity of our approach is questionable if the charged vortices become immobile. There is experimental evidence that this happens below a crossover temperature $T_{f}$, which increases with doping and reaches about $16 \mathrm{~K}$ in $\mathrm{La}_{2-x} \mathrm{Sr}_{x} \mathrm{CuO}_{4}$ for $x \approx x_{c}$ and falls off again for larger $x$ [22, 18]. $T_{f}$ is sketched in Fig. 3. Below this temperature the holes (charged vortices) form a glass and their dynamics strongly slows down. The experimental Néel temperature in this region should depend strongly on the time scale of the experiment. On the other hand, the true phase transition is governed by the behavior in the limit of infinite time so that the formation of a glass below $T_{f}$ should affect it only weakly. The doping dependence of $T_{f}$ can be qualitatively understood in our picture: In the magnetically disordered phase the logarithmic part of the interaction of charged vortices is screened on the length scale of the correlation length [2,3, 15], which is still large close to $T_{N}$. Thus the interaction of charged vortices (holes) changes smoothly at the transition and decreases for larger doping, leading to similar behavior of the freezing temperature $T_{f}$.

There are theoretical indications that the holes may form one-dimensional stripes at low temperatures [23]. Modeling stripes by a phenomenological anisotropic Heisenberg model, Castro Neto and Hone [24 calculated the Néel temperature $T_{N}(x)$ within a renormalizationgroup scheme and found good agreement with experiment. However, in this theory the critical concentration $x_{c}$ is basically a free parameter. Note, stripes formed by charged vortices consist of alternating vortices and antivortices, in order to lower the interaction energy. These stripes are automatically anti-phase domain walls 25, which are observed experimentally.

In conclusion, by starting from the assumption that holes doped into the Mott-Hubbard antiferromagnet dress with vortices and using independently obtained values for the exchange interaction and the antiferromagnetic correlation length in the ordered phase, we obtain a doping-temperature phase diagram for the antiferromagnetic phase in qualitative agreement with experiment. In particular, the predictions for the critical doping at zero temperature and the Néel temperature at zero doping are of the observed order of magnitude. Our approach uses a generalized BKT theory, which does not depend on any particular microscopic model. With an appropriate choice of the core energy of thermally created vortices we can obtain quantitative agreement with experiment. The core energy controls the shape of the phase boundary at small doping, but does not affect the region of higher doping, where the Néel temperature approaches zero at a critical hole density that is univer- sal in natural units. Our results show that stripes are not required to understand the data. The success of this theory based on vortex fluctuations emphasizes the importance of two-dimensionality for understanding the cuprates. This should also hold in the more strongly doped superconducting region. It would be desirable to include the spin rearrangement around holes, which is induced by frustration due to singlet formation, into an electronic theory of underdoped cuprates. For the antiferromagnetic phase such a theory should yield results similar to the ones shown here.

[1] G.M. Luke et al., Phys. Rev. B 42, 7981 (1990).

[2] V.L. Berezinskii, Sov. Phys. JETP 34, 610 (1972); J.M. Kosterlitz and D.J. Thouless, J. Phys. C 6, 1181 (1973); J.M. Kosterlitz, ibid. 7, 1046 (1974).

[3] P. Minnhagen, Rev. Mod. Phys. 59, 1001 (1987).

[4] V.J. Emery, Phys. Rev. Lett. 58, 2794 (1987).

[5] J. Schmalian, G. Baumgärtel, and K.H. Bennemann, Solid State Commun. 86, 119 (1993); G. Baumgärtel, J. Schmalian, and K.H. Bennemann, Phys. Rev. B 48, 3983 (1993).

[6] A. Aharony et al., Phys. Rev. Lett. 60, 1330 (1988).

[7] D. Coffey, K.S. Bedell, and S.A. Trugman, Phys. Rev. B 42, 6509 (1990); D. Coffey, T.M. Rice, and F.C. Zhang, ibid. 44, 10112 (1991).

[8] J.A. Vergés et al., Phys. Rev. B 43, 6099 (1991).

[9] M. Berciu and S. John, Phys. Rev. B 59, 15143 (1999).

[10] G. Seibold, Phys. Rev. B 58, 15520 (1998).

[11] W. Zhang, M. Avignon, and K.H. Bennemann, Phys. Rev. B 45, 12478 (1992), and references therein.

[12] N.E. Bickers, D.J. Scalapino, and S.R. White, Phys. Rev. Lett. 62, 961 (1989); N.E. Bickers and S.R. White, Phys. Rev. B 43, 8044 (1991); D.J. Scalapino, Phys. Reports 250, 329 (1995).

[13] A logarithmic long-range hole interaction is a possible origin of non-Fermi-liquid behavior, which is observed in underdoped cuprates [P. Kopietz (private communication)].

[14] C. Timm, S.M. Girvin, and H.A. Fertig, Phys. Rev. B 58, 10634 (1998).

[15] C. Timm, Physica C 265, 31 (1996).

[16] B. Keimer et al., Phys. Rev. B 46, 14034 (1992).

[17] F. Borsa et al., Phys. Rev. B 52, 7334 (1995).

[18] C. Niedermayer et al., Phys. Rev. Lett. 80, 3843 (1998).

[19] J.L. Tallon et al., Phys. Rev. B 51, 12911 (1995).

[20] G. Burns, High Temperature Superconductivity (Academic, Boston, 1992).

[21] C.J. Peters et al., Phys. Rev. B 37, 9761 (1988); T. Thio et al., ibid. 38, 905 (1988).

[22] R.J. Gooding, N.M. Salem, and A. Mailhot, Phys. Rev. B 49, 6067 (1994), and references therein.

[23] S.R. White and D.J. Scalapino, cond-mat/9907375, and references therein.

[24] A.H. Castro Neto and D. Hone, Phys. Rev. Lett. 76, 2165 (1996).

[25] C. Timm and K.H. Bennemann, J. Low Temp. Phys. 117, 205 (1999). 